\title{
Role of Vacancies in the Adsorption of Quantum Noble Gases inside a Bundle of Carbon Nanotubes
}

\author{
M. C. Gordillo \\ Departamento de Sistemas Físicos, Químicos y Naturales, Facultad de Ciencias Experimentales, Universidad Pablo de Olavide, \\ Carretera de Utrera, $\mathrm{km}$ 1, 41013 Sevilla, Spain \\ (Received 3 March 2006; published 30 May 2006)
}

\begin{abstract}
Diffusion Monte Carlo calculations on the ${ }^{4} \mathrm{He}$ and ${ }^{20} \mathrm{Ne}$ adsorption in the interstices of a bundle of $(10,10)$ carbon nanotubes are reported. The results indicate that the presence of a carbon vacancy is enough, at least in some cases, to impede the adsorption of quantum gases in those systems. This could explain some discrepancies between the experimental data and the theoretical calculations about the possibility of adsorption of gases inside a bundle of carbon nanotubes.
\end{abstract}

PACS numbers: $68.90 .+\mathrm{g}, 05.30 .-\mathrm{d}$

Carbon nanotubes have been the focus of a lot of attention since their discovery in 1991 by Iijima [1]. Among their many interesting properties is their purported gas adsorption capability, both inside the open-ended tubes and in the outer surface of the association of tubes commonly termed a bundle (see, for instance, Ref. [2], and references therein). There is, however, a third possibility: the insertion of small species $\left({ }^{4} \mathrm{He}, \mathrm{H}_{2}\right.$, and $\left.\mathrm{Ne}\right)[3]$ in the triangularly shaped interstices (IC's) existing in between every three carbon nanotubes forming a bundle. The adsorption in these places would increase appreciably the uptake of the bundles, making them interesting as gas reservoirs. Despite some positive indications to the contrary [4-6], there are experimental results suggesting there is no such adsorption $[7,8]$.

In any case, the mere possibility has been extensively studied from the theoretical point of view [2]. Summarizing, we can say that, in the case of the species indicated above the calculations support adsorption, both in the outer surfaces and inside the interstices of the bundles [3]. The case of $\mathrm{Ne}$ has been considered in detail in Ref. [9] in comparison to the experimental results of Talapatra et al. [7]. It was found that, under the approximations used in the calculation, neon should have entered the IC's of a bundle of $(10,10)$ carbon nanotubes, something denied by the experimental results. The same can be said of the more recent experimental data for ${ }^{4} \mathrm{He}$ [8]: They contradict the theoretical calculations indicating helium adsorption inside the IC's [10].

A possible way out of this discrepancy is the one indicated in Ref. [11] for the case of $\mathrm{H}_{2}$. It is shown there that, for some values of the adsorbate-nanotube interation parameters, the radius of the tube, and the intertube distances, there is a sizable minimum in the potential energy just outside the entrance of the IC. That would make the hydrogen to stay there instead of entering the IC, effectively closing the rest of the interchannel to further adsorption. Even though this is a possibility for the case of $\mathrm{H}_{2}$, the effect seen depends heavily on the $\mathrm{H}_{2}-\mathrm{C}$ interaction parameters, not known exactly, and on the tube radius. The goal of the present work is to consider other possibilities that accounted for the observed lack of adsorption of all quantum species inside the IC's of a bundle of carbon nanotubes, basically corrugation and the possible existence of defects.

To do so, diffusion Monte Carlo (DMC) calculations of ${ }^{4} \mathrm{He}$ and ${ }^{20} \mathrm{Ne}$ inside both a $(5,5)$ carbon nanotube and an IC in between three $(10,10)$ tubes were carried out. A DMC calculation is a standard technique to solve the Schrödinger equation in order to obtain the ground state of the considered system [12] a priori. In all cases, the trial function needed in the method was a product of Jastrow-type functions [12], with the product extending to all the C-gas pairs. The total carbon-nanotube-gas potential was the result of summing up all the individual $\mathrm{C}$-quantum species interactions, with the same value for all carbon atoms (C-He interaction from Ref. [13] and $\mathrm{C}-\mathrm{Ne}$ one from Ref. [3]). Both potentials are of the Lennard-Jones type and are widely used in the literature, even though their parameters are admitted to have an uncertainty of about a 15\% [3], an amount not enough to change the results presented here. The length of the simulation cells was $49.2 \AA$. A single atom of the gas was placed at $z=0$ (the beginning of the tube or of the IC) and left to evolve until no appreciable change in the density profiles (see below) was found.

Carbon nanotubes are through to have a certain amount of defects $[6,14,15]$, being single carbon vacancies among the simplest of them [16-19]. In principle, the removing of a single carbon atom should leave three dangling bonds. However, it is not clear if such an arrangement is stable [18], as it seems to be the so-called 5-1DB defect. This is the result of two of the dangling bonds recombining with each other to form a pentagon and leaving the third one unchanged. There are two forms of doing this, termed symmetric and asymmetric. It appears that for $(n, n)$ nanotubes the last one is the most stable [16,19]. To consider the influence of vacancies in the adsorption of quantum gases, every of these arrangements (three dangling bonds and the two types of 5-1DB defects) were placed in the center of 


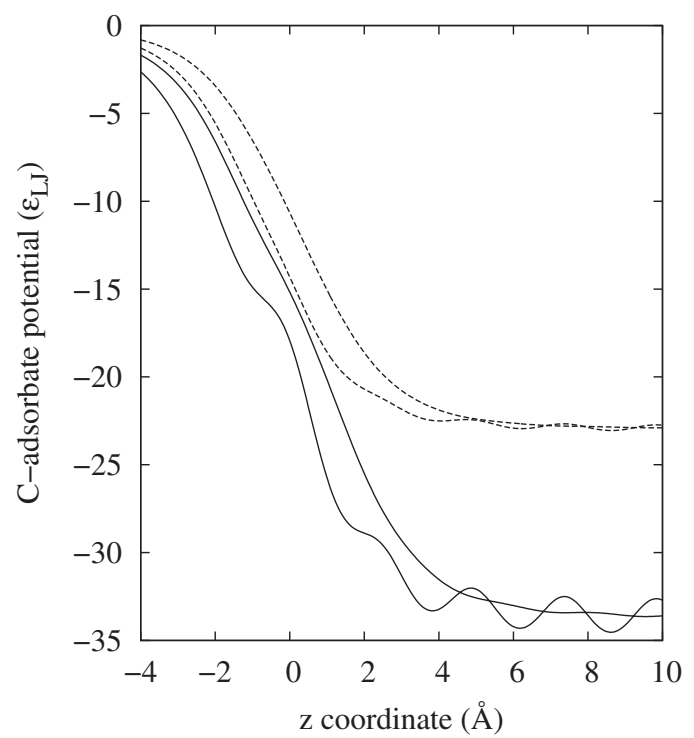

FIG. 1. Potential profiles for the carbon-nanotube-gas interaction inside an IC of a $(10,10)$ bundle in units of the LennardJones parameter $\epsilon$. Solid lines correspond to $\mathrm{Ne}$ and dashed ones to He. Two cases are shown: that of the maximum (wavier) and minimum (smoother) corrugation. The beginning of the tube is at $z=0$. The $x$ and $y$ coordinates are fixed to coincide with the geometrical center of the IC.

the simulation cell. Periodic boundary conditions were not considered.

Figure 1 is shown to give us an idea about the corrugation effects that an atom will see inside an interstice of a bundle of carbon nanotubes. It represents the potential energy for a single atom at the geometrical center of the IC for different values of the $z$ coordinate in $\epsilon_{\mathrm{LJ}}$ units. However, in the simulations, the quantum species were not confined to this single point in the $x y$ plane, but they were allowed to move freely. The IC starts at $z=0$, negative values of this coordinate corresponding to locations out of the IC. We have the case of $\mathrm{He}$ (top) and $\mathrm{Ne}$ (bottom). The three tubes are considered to be open (with their caps removed), located at a minimum distance between their centers of $17 \AA$, and perfectly aligned (starting all of them at $z=0$ ). According to Ref. [11], these are the most propitious conditions for gas adsorption in the IC's, so we can concentrate in other hindering effects. The smoother curves correspond to the situation in which the tubes are displaced $0.82 \AA$ with respect to each other, i.e., $1 / 3$ of the length of the unit cell of a $(n, n)$ carbon nanotube $(2.46 \AA)$. To our knowledge, this is the case in which the corrugation effects are smaller. The other set of curves represents the case in which the three tubes are perfectly aligned, with every carbon ring located at the same $z$ coordinate as the corresponding two neighboring rings. This will be the case with the greater corrugation. One would expect these to be the limiting cases for all the properties presented here. In any case, there is something
TABLE I. Binding energies in $\mathrm{K}$ for a single atom inside an interstice of a bundle of $(10,10)$ nanotubes for different corrugations.

\begin{tabular}{llc}
\hline \hline & \multicolumn{1}{c}{${ }^{4} \mathrm{He}$} & ${ }^{20} \mathrm{Ne}$ \\
\hline Maximum corrugation & $322.853(15)$ & $973.28(5)$ \\
Minimum corrugation & $317.110(15)$ & $964.30(3)$ \\
Smoothed potential & $323.41(1)[10]$ & $1011.15(2)[9]$ \\
\hline \hline
\end{tabular}

immediately apparent: There is no minimum outside the interchannels in any case; i.e., the gas should readily enter the IC's. On the other hand, the corrugation effects could be sizable for Ne. This is confirmed by the results given in Table I: There we can find the binding energies of the gas species inside the nondefective IC for the two limiting corrugation cases together with the previous results for the cylindrically averaged potentials with the same parameters.

The effect of the presence of a carbon vacancy in the global potential is displayed in Fig. 2. The Ne case is shown for the least corrugation and with the most stable type of defects (asymmetric 5-1DB), We can see that the size of the disturbance is about $2 \sigma_{\mathrm{LJ}}$ in length and $\epsilon_{\mathrm{LJ}}$ in energy. This is typical of all arrangements.

To isolate the effect of the presence of a carbon hole of those of the corrugation and orientation of the vacancy with respect to the center of the interchannels, we performed DMC calculations of the adsorption of ${ }^{4} \mathrm{He}$ and ${ }^{20} \mathrm{Ne}$ inside a $(5,5)$ nanotube. The radius of the tube is $3.42 \AA$, of the same order as the minimum distance between the center of

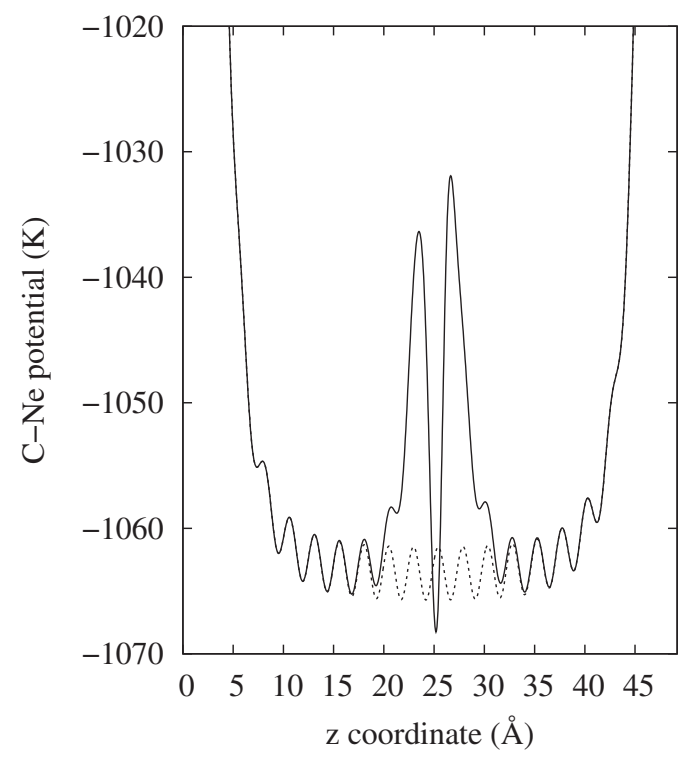

FIG. 2. Same as in Fig. 1 but only for the case of Ne and the smoother corrugation. The dashed line indicates an interstice made of three perfect tubes, while the solid line is the interaction in presence of an asymmetric 5-1DB defect in one of the tubes as close as possible to the geometrical center of the IC. 


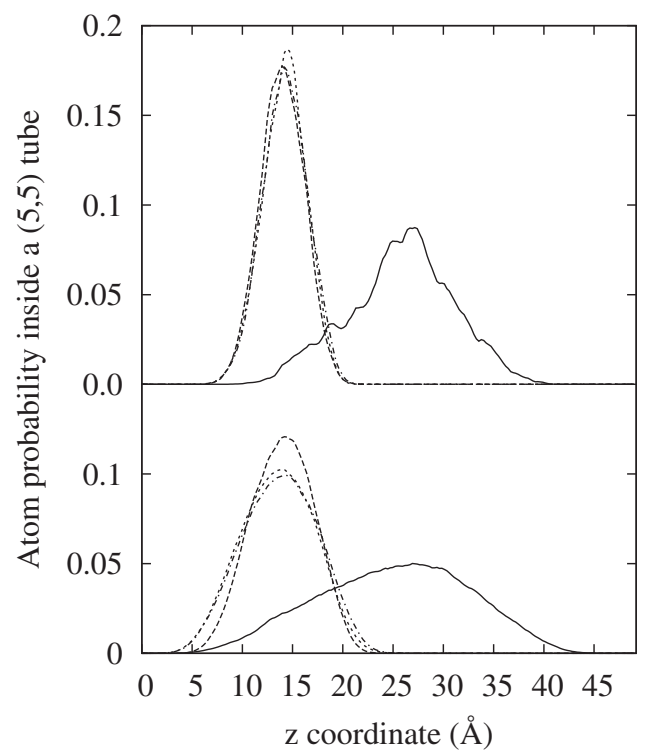

FIG. 3. Probability density of having a single atom inside a given $z$ position in a $(5,5)$ nanotube. We present here the case of $\mathrm{Ne}$ (top) and ${ }^{4} \mathrm{He}$ (bottom). Dashed-dotted lines indicate the result for a perfect tube, solid lines what we obtain when an asymmetric 5-1DB is introduced, dashed lines the situation for a symmetric defect of the same type, and the dotted curves are the probabilities for an hypothetical three dangling bond vacancy.

the IC and the closest wall (around $3 \AA$ ). This is a cylindrical environment in which the effects of corrugation are pretty similar to what we have seen in the smoother cases in Fig. 1. In Fig. 3, we have the results for ${ }^{4} \mathrm{He}$ (bottom) and ${ }^{20} \mathrm{Ne}$ (top). It can be easily seen that, for the nondefective tubes, the gas species distributes more or less uniformly along the simulation cell, while the presence of any kind of vacancy effectively prevents it from passing to the other half of the simulation cell. We can also see that the range explored by the adsorbate increases from top to bottom. This is due to the fact that the Lennard-Jones $\sigma$ parameter for the C-gas interaction decreases from $\mathrm{Ne}$ to $\mathrm{He}$.

With the above results in mind, we can look at what happens to helium inside an IC. In Fig. 4, the solid line indicates the results for the most corrugated system, while the dashed-dotted line is also for a defect-free interchannel but with minimum corrugation. Obviously, the helium atom roams the available space freely enough in both cases; corrugation seems not to be an issue here. However, the situation changes when a vacancy is considered. Given the essential equivalence of all defects deduced from the results already displayed in Fig. 3, only the most stable ones (the asymmetric 5-1DB's) were considered. The dashed line indicates the atom probability when the missing carbon atom is located as close as possible to the center of the interchannel in the least corrugated arrangement: The helium atom passage to the other part of the tube is forbidden. On the other hand, one might ask what would be the situation in which the presence of a carbon vacancy

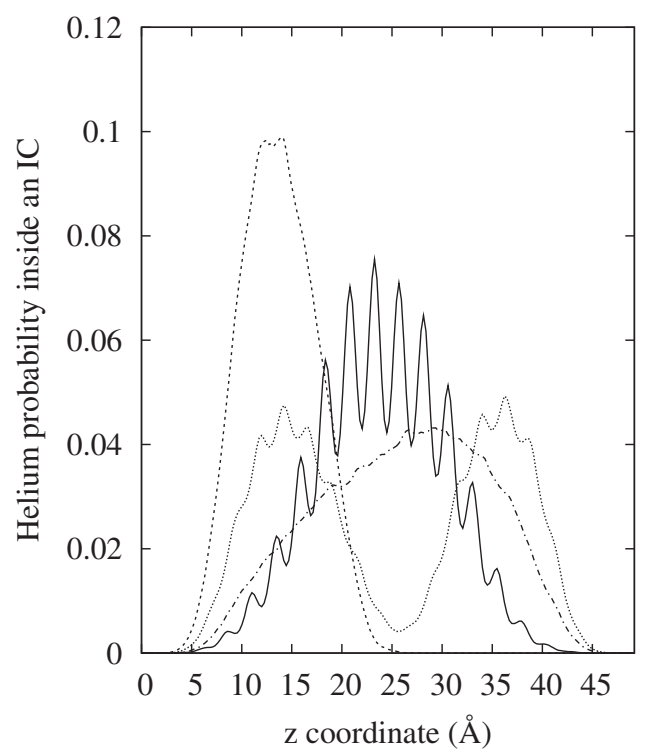

FIG. 4. Atomic density profile for helium inside an IC. Solid (dashed-dotted) line, ${ }^{4} \mathrm{He}$ inside a defect-free IC with the maximum (minimum) corrugation; dashed (dotted) line, results for an asymmetric 5-1DB vacancy closest to (furthest from) the center of the IC for the minimum corrugation cases.

is less likely to influence the helium movement. Considering that inside a bundle a carbon nanotube is part of six IC's, one might conclude that the farthest a hole can be from the IC axis is $30^{\circ}$ in each direction. In that situation (dotted line), we observe two maxima in a roughly symmetrical atom distribution with a minimum in between corresponding to the $z$ coordinate of the vacancy. This means that the influence of the carbon hole does not preclude the helium atom to move around it. When the deviation of the vacancy from the axis is $20^{\circ}$, the helium movement is also impeded. This would mean that about $2 / 3$ of the single vacancies in a tube could severely impede the helium movement.

The behavior of Ne inside an IC is slightly different from that of He. Figure 5 displays it for maximum (solid line) and minimum (dotted line) corrugations. We can see its behavior is different from helium, since at least in some cases, the corrugation affects the atom movement enough to stop it. The dashed line shows what happens when a $5-1 \mathrm{DB}$ defect located at $30^{\circ}$ away from the IC axis. The perturbation caused by any hole is big enough to confine the $\mathrm{Ne}$ atom to its original half part of the simulation cell.

One can think of several remaining issues concerning the calculations presented here. First, to our knowledge, there is no study in the literature about concerning the carbon-noble gas potential of the atoms surrounding the vacancy. Here we used the same value as for the other carbon atoms, since this is a kind of worst-case scenario. Since both helium and neon are noble gases, one would expect a physisorption interaction reasonably described by a Lennard-Jones potential. If this potential is less attractive, 


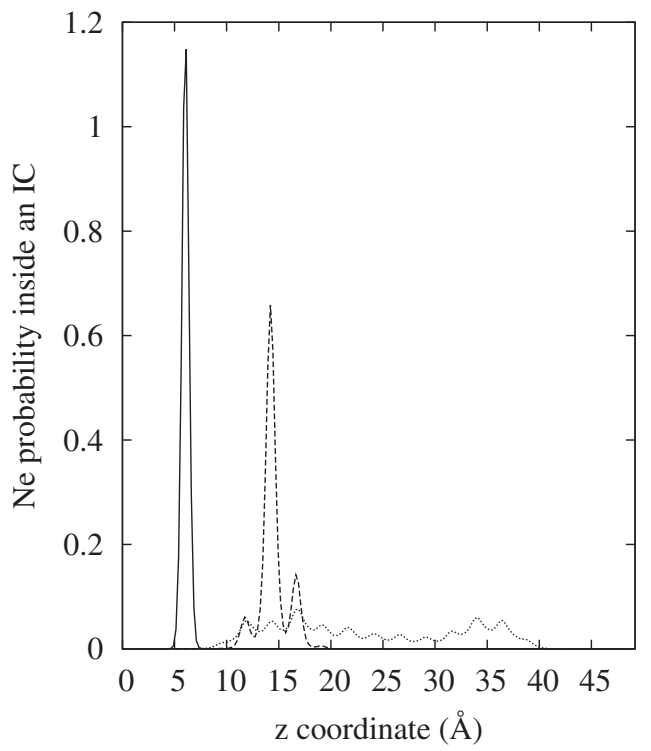

FIG. 5. Same as in Fig. 4 but for neon inside an IC. The solid (dotted) line represents neon inside a defect-free IC with the maximum (minimum) corrugation, while the dashed line is results for an asymmetric 5-1DB vacancy farthest from the center of the IC and in the case of minimum corrugation.

the barriers should be greater than that in the present study. On the other hand, a more attractive interaction could create a potential well instead of a barrier. That potential well would trap an adsorbate atom that would create an even bigger barrier. In any case, the adsorption of more external atoms would stop.

Second, the technique used, a DMC calculation, implies that the results presented here are strictly valid only at $0 \mathrm{~K}$. However, since we are in quasi-one-dimensional systems, one can approximate very well the thermal behavior of the system by that of a 1D free particle [9]. A free particle in the external potential already discussed stops close to the defect when the temperature is about $1 / 10$ of the energy barrier. This means $\sim 1.5-2 \mathrm{~K}$ for the case of helium and 3-4 K for Ne. For higher temperatures, the hole does not stop the atom movement. However, there is still a kinetic effect to take into consideration. An very simple analysis on the lines of those of Refs. [11,20] indicates that below $\sim 3 \mathrm{~K}$ in the first case and $\sim 6 \mathrm{~K}$ in the second, the overcoming of the barrier will be very slow. One should take also into account [11] that the adsorption takes place only through the edges of the IC, which makes the process even slower. This could explain the lack of adsorption found in Ref. [8], where the temperature was $2.5 \mathrm{~K}$. However, this is not an explanation for the case of $\mathrm{Ne}$, when the experimental temperatures were up to $60 \mathrm{~K}$ [7]. Within the assumptions made in this Letter, one would have to resort to at least two defects relatively close together to form a potential barrier high enough to stop Ne from adsorbing.

Summarizing, DMC calculations support the idea that carbon vacancies in the graphene network of a carbon nanotube could severely impede the movement of adsorbates inside narrow environments. The presence of vacancies could be, at least in some cases, the cause of the lack of adsorption of quantum gases inside a bundle of carbon nanotubes found experimentally.

The Spanish Ministry of Education and Science (MEC) and the Junta de Andalucia are thanked for financial support under Grant No. BFM-2003-08211-C03-01 and group FQM-205, respectively.

[1] S. Iijima, Nature (London) 354, 56 (1991).

[2] M. M. Calbi, M. W. Cole, S. M. Gatica, M. J. Bojan, and G. Stan, Rev. Mod. Phys. 73, 857 (2001).

[3] G. Stan, M. J. Bojan, S. Curtarolo, S. M. Gatica, and M. W. Cole, Phys. Rev. B 62, 2173 (2000).

[4] A. C. Dillon, K. M. Jones, T. A. Bekkedahl, C. H. Kiang, D. S. Bethune, and M. J. Heben, Nature (London) 386, 377 (1997).

[5] W. Teizer, R. B. Hallock, E. Dujardin, and T. W. Ebbesen, Phys. Rev. Lett. 82, 5305 (1999).

[6] W. Teizer, R. B. Hallock, E. Dujardin, and T. W. Ebbesen, Phys. Rev. Lett. 84, 1844 (2000).

[7] S. Talapatra, A.Z. Zambano, S.E. Weber, and A.D. Migone, Phys. Rev. Lett. 85, 138 (2000).

[8] J. V. Pearce, M. A. Adams, O. E. Vilches, M. R. Johnson, and H. R. Glyde, Phys. Rev. Lett. 95, 185302 (2005).

[9] M. C. Gordillo, L. Brualla, and S. Fantoni, Phys. Rev. B 70, 245420 (2004).

[10] J. Boronat, M. C. Gordillo, and J. Casulleras, J. Low Temp. Phys. 126, 199 (2002).

[11] M. M. Calbi and J.L. Riccardo, Phys. Rev. Lett. 94, 246103 (2005).

[12] J. Boronat and J. Casulleras, Phys. Rev. B 49, 8920 (1994).

[13] G. Stan and M. W. Cole, Surf. Sci. 395, 280 (1998).

[14] D. B. Mawhinney, V. Naumenko, A. Kuznetsova, J. T. Tates, Jr., J. Liu, and R.E. Smaley, Chem. Phys. Lett. 324, 213 (2000).

[15] E. Dujardin, T. W. Ebbesen, A. Krishnan, and M. M. J. Treacy, Adv. Mater. 10, 611 (1998).

[16] A. J. Lu and B. C. Pan, Phys. Rev. Lett. 92, 105504 (2004).

[17] P. M. Ajayan, V. Ravikumar, and J. C. Charlier, Phys. Rev. Lett. 81, 1437 (1998).

[18] A. V. Krasheninnikov, K. Nordlund, M. Sirviö, E. Salonen, and J. Keinonen, Phys. Rev. B 63, 245405 (2001).

[19] S. Zhang, S. L. Mielke, R. Khare, D. Troya, R. S. Ruoff, G. C. Schatz, and T. Belytschko, Phys. Rev. B 71, 115403 (2005).

[20] F. M. Bulnes, V.D. Pereyra, and J.L. Riccardo, Phys. Rev. E 58, 86 (1998). 